\title{
Imaging and Genomic Classification of Brain Alteration Induced By Gustatory Bitter Stimulus, A Pilot Study
}

\author{
Luigi Barberini ${ }^{1 *}$, Iole Tomassini Barbarossa ${ }^{2}$, Melania Melis ${ }^{2}$, Francesco Marrosu ${ }^{1}$, Paolo Siotto ${ }^{3}$ and \\ Luca Saba $^{1}$ \\ ${ }^{1}$ Department of Medical Sciences and Public Health, Italy \\ ${ }^{2}$ Department Biomedical Sciences, Italy \\ ${ }^{3}$ Department Diagnostic Imaging, G. Brotzu Hospital, Italy
}

*Corresponding author: Luigi Barberini, Department of Medical Sciences and Public Health, Italy

ARTICLE INFO

Received: 幽 January 16, 2020

Published: 豐 January 23, 2020

Citation: Luigi Barberini, Iole Tomassini Barbarossa, Melania Melis, Francesco Marrosu, Paolo Siotto, Luca Saba. Imaging and Genomic Classification of Brain Alteration Induced By Gustatory Bitter Stimulus, A Pilot Study. Biomed J Sci \& Tech Res 24(5)-2020. BJSTR. MS.ID.004120.

Keywords: Gustatory Pathways; Bitter Taste; fMRI; Default Mode Network

Abbreviations: PROP: Propylthiouracil; fMRI: Functional Magnetic Resonance Imaging; ROIs: Regions of Interest; DMN: Default Mode Network; PCC: Posterior Cingulate Cortex; MPFC: Medial Prefrontal Cortex; RLP: Right Lateral Parietal Cortices; ICC: Intrinsic Connectivity Contrast

\section{ABSTRACT}

Objectives: Despite the exhaustive information concerning the role of physiological structures involved in gustatory stimuli, there is still a need to clarify as to whether the gustatory sense is processed in the human brain and how different kind of gustatory stimuli affect brain areas involved in taste processing. To characterize the networks that can be involved in shaping the properties of the gustatory pathways.

Materials and Methods: In this fMRI study we investigated two populations characterized by their opposite response (sensitive/non-sensitive) to bitter gustatory stimulus induced by propylthiouracil molecule (PROP). In the MRI experiment PROP was delivered to the subjects by an impregnated filter paper disk. The individuals recruited for this study are 5 super-testers and 8 non-testers to the bitter stimulus. The procedure defines two different groups of subjects "non-taster" and super-taster" which take part to the fcMRI experimental stud Functional data, acquired by a GE Medical Signa 1.5Tesla HD Optima Scanner, were processed with SPM12 toolbox and network pathways were analysed using the CONN toolbox. The general linear model (GLM) technique was applied by allowing for statistical evaluation of activation. Low oscillation of Bold signals [0.09:0.9 Hz] time series were processed by calculating the semi partial correlation index between voxels; the integrated connectivity contrast measure was applied for connectivity map calculation. All contrasts were examined with a voxel wise t-test ( $p<0.05$ uncorrected). We examined activations using the four hubs of the Default Mode Network of the brain, as crucial seeds for the connectivity analysis. A dissimilarity contrast matrix between non-taster e super-tester subjects (between-subjects contrast) and between the two conditions of the bitter stimulus submission and no stimulus submission adopted in the fMRI experiment was created. DMN ROIs projection towards the Brodmann areas were detected with t-test significance test.

Results: In our fMRI study the areas of Insula, Cingulate Cortex, Somatosensory Associative Cortex, Entorhinal Cortex, Premotor Cortex Area are correlated to the four DMN areas during the bitter stimuli processing more in super tester than in the control population.

Discussion: The bitter taste is differently processed by the two groups of supertasters and non-testers and our results suggests further tests, increasing the population and making different hypothesis in order to encompass the main gustatory pathways and their correlations and differentiation in processing and interpreting gustatory information.

Conclusion: It seems likely that further studies are needed to address the possible reasons of this difference between these population. A working hypothesis can privilege an evolutionary differentiation between these two populations, as the correct appreciation of bitter taste can be an advantageous step in peering through possible dangerous tastes in a primitive setting. 


\section{Introduction}

Bitter taste sensitivity varies greatly among individuals, and since many bitter substances are toxic, the genetic ability to taste the bitter quality confers an important survival advantage by allowing super-taster individuals, compared to non-tasters, to recognize potentially harmful substances. The best-known example of the bitter taste variability is the genetic ability to taste the bitter thiourea compounds, such as 6-n-Propylthiouracil (PROP) [1]. PROP sensitive and non-sensitive individuals are defined as tasters and non-tasters. The term "super-taster" is used to distinguish individuals who perceive PROP as extremely bitter from those who perceive it as moderately bitter. The allelic diversity of the gene codifying for TAS2R38 receptor can explain most phenotypic differences in PROP tasting [2,3]. Several investigations have attempted to elucidate the molecular basis and the genetic and psychophysical features of this human trait. Some studies have consistently reported that PROP super-tasters have a higher density of fungiform papillae on the anterior tongue surface, suggesting that they are also anatomically distinct from the other PROP taster groups in their peripheral taste system.

Although human brain pathways involved in the complex gustatory circuitry are well known, there is still the need to elucidate how taste signals are processed in the brain and whether stimuli may influence, in the neural processing, different brain areas depending on the meaning that they have for individuals. We present data of a pilot study of Functional Magnetic Resonance Imaging (fMRI) aimed at determining if individual differences of taste perception of PROP can be represented in the brain by different activation patterns. The four major Regions of Interest (ROIs) constituting the Default Mode Network (DMN), e.g. the Posterior Cingulate Cortex (PCC), the Medial Prefrontal Cortex (MPFC), and left and Right Lateral Parietal Cortices (LLP and RLP), were examined as sources for the connectivity analysis [4]. Targets were the complete set of Broadman area.

\section{Material and Methods}

\section{Subjects}

13 ( 2 males, 11 females, mean age $31.6 \pm 10.9$ y) caucasian non-smoker subjects were recruited according to standard procedures. They were genotyped for TAS2R38 gene according to Cossu Movement Disorder [5], Melis Laryngoscope [6], and classified for their PROP taster status according to [7] and validated in several studies [8,9]. Five subjects were classified as PROP super-tasters and had a homozygous genotype for the taster receptor variant (PAV/PAV), while the others were classified as non-tasters and had homozygous genotype for the non-taster receptor variant (AVI/ AVI).

\section{Image Acquisition}

Images acquisition was performed on a GE Medical Signa 1.5Tesla HD Optima Scanner (Milwaukee, USA). Structural 3D
Image sequence was acquired with the 3DFSE SAGITTAL 3D CUBE with a repetition and echo time of TR/TE 2987/97.5ms. Functional images were acquired with EPI protocol. The Sequence "Functional Connect" was created with TR/TE 2624/45 ms 64x64 FA 90 FOV $=250 \times 250 \mathrm{~mm}$; slice thickness was $5 \mathrm{~mm}$ with 2-mm gap. Head Brain Coil used was the 8HRBRAIN high-resolution 8-channel brain coil.

\section{Experimental Protocol}

Subjects underwent one fMRI run in the scanner. During the fMRI run they were requested to stay firm with relaxed muscles and their eyes closed. For each subjects the fMRI run included a $15 \mathrm{~min}$ recording of the brain activity at rest followed by another $15 \mathrm{~min}$ recording of the activity induced by PROP bitter taste stimulation. PROP stimulation was performed by placing a filter paper disk impregnated with $50 \mathrm{mmol} / \mathrm{L}$ of the compound on the tip of the tongue. Taste stimulation induces brain activity revealed by fMRI.

\section{Data Analysis}

Data were processed with the SPM12 software for the general linear model $(G L M) Y=X \beta+\varepsilon$ [10]. Following spatial-temporal adjustment of the volumes, the GLM technique allowed for statistical evaluation of activation. All contrasts were examined with a voxel wise significance level of $\mathrm{p}<0.05$ (t-test) corrected for multiple comparisons across the brain volume. Minimum cluster size was settled at 20. The t-test was used to compare simultaneously differences between super-tasters and non-tasters, and rest and stimulation condition. As previously reported, BOLD signals from voxels of interest can be processed in CONN software using several indices of connectivity [11]. Connectivity measure is performed at the voxel-to-voxel level and, in order to discuss connectivity properties in connection with spatially segregated brain functions, a seed to voxel, and ROI to ROI analysis can be performed. In this experiment, brain areas of interest were spatially labelled as in Broadmann Areas (BA) in order characterize the source regions for the extraction of the time series of interest. The same areas were subsequently used as labels for targets in ROI-to-ROI, and seed to voxel analysis. Data presented in this paper focuses on the zero-lagged bivariate-correlation linear measure of functional connectivity between two sources defined as a bivariate correlation:

$$
r=\left(X^{t} \cdot X\right)^{1 / 2} \cdot b \cdot\left(\left(Y^{t} \cdot Y\right)^{-1 / 2}\right.
$$

This is the Voxel-Level Functional Connectivity MRI measures derived from the Voxel-to-Voxel Connectivity Matrix r(x,y); in this project the strength of the global connectivity pattern between each voxel and the rest of the brain with the Intrinsic Connectivity Contrast (ICC) was characterized. ICC is defined as:

$$
I C C=\frac{1}{|\Omega|} \sum_{y=\Omega} r=(x, y)^{2}
$$

ICC represents the average $r^{2}$ connectivity of a given voxel $i$ and all the other voxels, and the power map calculated provides 
a metric that requires no a priori information either in terms of arbitrary thresholds or ROI definitions [12]. We used the ICC metric to produce intrinsic connectivity contrast maps because this metric does not need a priori knowledge for defining any ROIs and ICC can be used as an exploratory tool for investigating the functional organization voxel by voxel; as reported in the formula, ICC is a L2 Norm. It is important to highlight that functional connectivity is modulated by the brain state. Our initial analyses compare voxel wise connectivity estimates within the Default Mode Network in a rest state condition. We supposed that regions within DMN should exhibit increased connectivity with other brain areas the network at rest compared with the state during the bitter solicitation, consistent with prior reports of elevated activation of DMN during rest [13]. For this reasons ICC seems to be best index of connectivity in this kind of experiment. Variations in this measure between different experimental conditions (or across patient populations) highlight the tissue elements in the brain that exhibit altered connectivity as a function of condition. ICC index provide explorative tools to localize changes in functional connectivity related to experimental modulations.

\section{Results}

Our tests were performed to prove the validity of the hypothesis of greater activation in some cortical areas generated by the bitter stimulus of Prop in the comparison between the "supertesters" and the "non-testers". The area in the brain functionally devoted to the gustatory stimulus process is identify in the frontal operculum area and in insular cortex. This area is identified as 43 Brodmann's area, which is activated in response to the generic pressure directly applied to the eardrum and to the indirect pressure generated, for example, by oral intake of the eating and drinking. Because eating and drinking change pressure on the middle ear and eardrum, Brodmann Area 43 may be a part of the primary somatosensory cortex activated during these processes related to the taste evaluation brain function. Brodmann area 43 was also functionally active with connections to the cortex of the frontal cerebellum. During our experiment we collected several other areas of the brain connected to BA (43) and represented graphically in the following Figure 1.

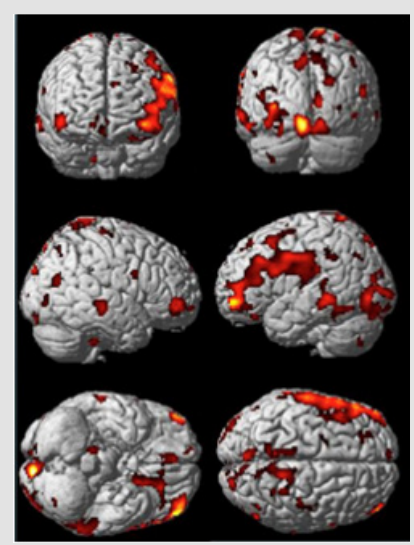

Figure 1: Differential Cortical activation in SPM style for comparison of non-tester vs super-tester.
From the data activation exhibited in the previous picture we can calculate the connectivity metric applying the SVD, singular value decomposition, method to the time serial covariance matrix of BOLD signals from the voxel and clusters of voxels. These data report a voxel mapping as report in the following picture. It is important highlight that correlation between these areas can be positive and negative. Data of connectivity calculated on the activation measures of the previous figure are exhibited in the Figure 2. Starting by the BA(43), the area involved in the taste processing, we can consider the connection towards several areas connected to the DMN, as the PCC (posterior cingulate cortex); this is the upper part of the "limbic lobe". The cingulate cortex is made up of an area around the midline of the brain. Surrounding areas include the retrosplenial cortex and the precuneus. Efforts to understand the functional architecture of the brain have consistently identified multiple overlapping large-scale neural networks that are observable across multiple states. Despite the ubiquity of these networks, it remains unclear how regions within these large-scale neural networks interact to orchestrate behavior.

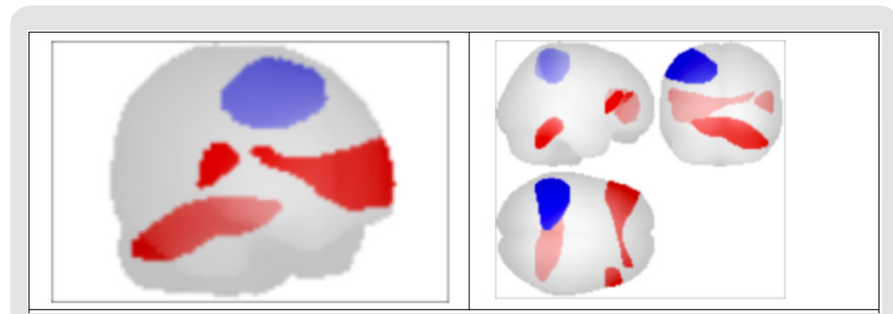

Figure 2: Voxel to Voxel mapping for correlations with positive and negative connectivity colors of areas (Blu = positive; red=negative).

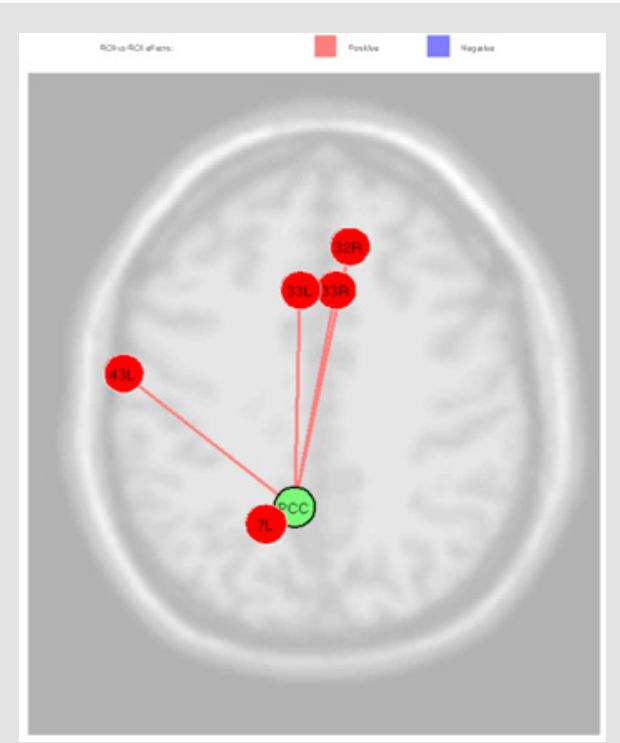

Figure 3: PCC connection in comparison of non-tester vs super-tester.

Given that the precuneus is a Functional Core of the Default-Mode Network our subjects are requested to do no tasks during fcMRI sessions, [14]. During the first part of the experiment they simply stay in idle, by standing still and trying to leave 
the mind wandering without a specific topic. In the second session of fcMRI they were requested to experiment the bitter taste of the propylthiouracil. In these conditions the BA (43) exhibit correlation with Anterior Cingulate Cortex (right and left BA33, the Subcentral Area, the Somatosensory Associative Cortex and Dorsal Anterior Cingulate Cortex Figure 3. The PCC source was directly correlated Anterior Cingulate Cortex (right) (beta 0.16; p=0.02), Anterior Cingulate Cortex (left) (beta 0.17; p=0.02), Subcentral Area "Gustatory Cortical Area" (right) (beta 0.16; p=0.03), Somatosensory Associative Cortex (left) (beta 0.13; $\mathrm{p}=0.03$ ) and Dorsal Anterior Cingulate Cortex (right) (beta 0.10; $\mathrm{p}=0.04$ ) Figure 4.

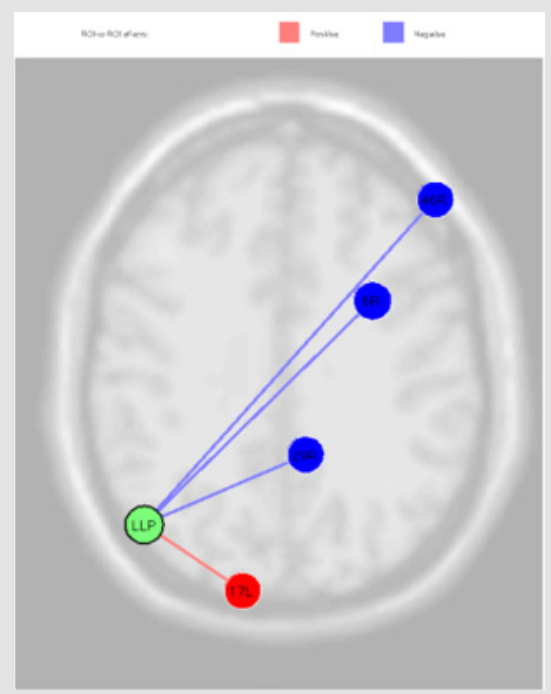

Figure 4: The connection of LLP source with Premotor Cortex (left) (beta $-0.14 ; \mathrm{p}=0.02$ ), Dorsolateral Prefrontal Cortex (right) (beta -0.05; $\mathrm{p}=0.02$ ) and Retrosplenial Cingulate Cortex (right) (beta $-0.13 ; \mathrm{p}=0.02$ ), and with Primary Visual Cortex V1 (left) (beta 0.13; $\mathrm{p}=0.04$ ).

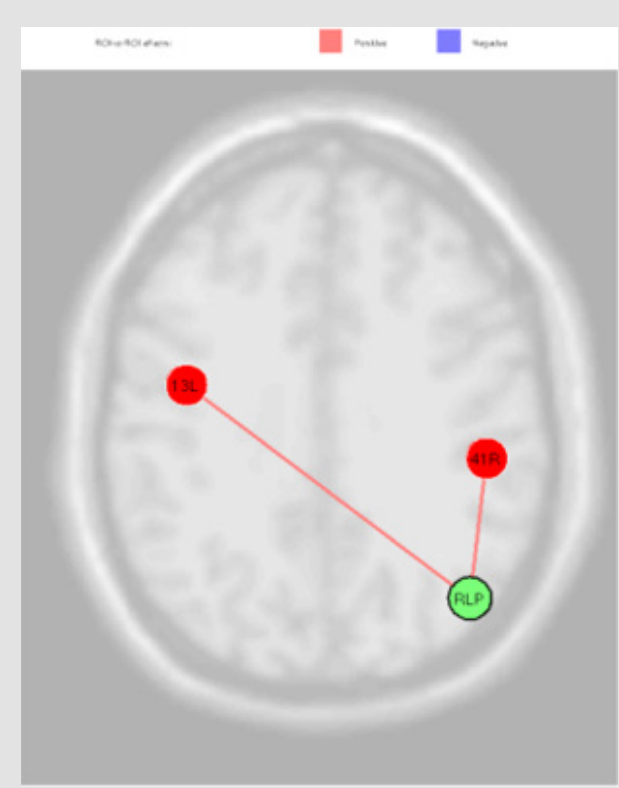

Figure 5: RLP source was directly correlated with Auditory Cortex (right) (beta 0.19; $\mathrm{p}=0.04$ ) and Insula Cortex (left) (beta $0.06 ; \mathrm{p}=0.04$ ).
The activation of LLP source, reported in the Figure 4, was inversely correlated with Premotor Cortex (left), Dorsolateral Prefrontal Cortex (right) and Retrosplenial Cingulate Cortex (right), and directly with Primary Visual Cortex V1 (left); the MPFC source was inversely correlated with Somatosensory Associative Cortex (right) and Anterior Entorhinal Cortex (right); the PCC source was directly correlated Anterior Cingulate Cortex (right), Anterior Cingulate Cortex (left), Subcentral Area (right), Somatosensory Associative Cortex (left) and Dorsal Anterior Cingulate Cortex (right); RLP source was directly correlated with Auditory Cortex (right) and Insula Cortex (left) Figure 5. Finally, we report the MPFC connections revealed in our experiments Figure 6.

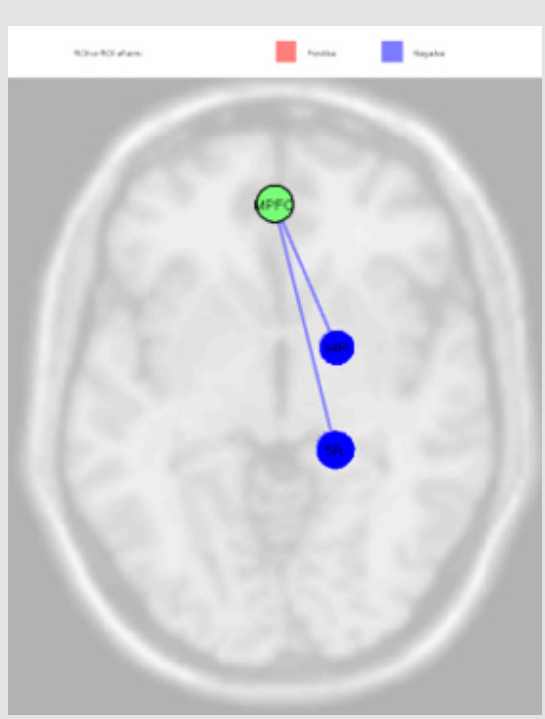

Figure 6: The MPFC source was inversely correlated with Somatosensory Associative Cortex (right) (beta -0.10; $\mathrm{p}=0.01$ ) and Anterior Entorhinal Cortex (right) (beta -0.10; $\mathrm{p}=0.03)$.

\section{Discussion}

We used the fcMRI and the genotyping procedures to study the putative brain pathways of taste processing. In doing this investigation we hypothesized that, since many bitter natural substances are toxic, there would be a genetic ability to taste the bitter compounds. Moreover, it is likely that such biological ability confers an important survival advantage by allowing supertaster individuals to recognize potentially harmful substances, when compared to non-tasters, [15]. Several investigations have attempted to elucidate the molecular basis as well as the genetic and psychophysical features of this specific trait. However, the genetic issue is by no means the only answer to the human behavior. The rapid development of brain imaging techniques, such the Nuclear Magnetic Resonance, spurred new methods of investigation aimed at better exploiting the modalities to visualize how the external signals are processed by the human brain. The wealth of recent studies on connectomics, a new science based on the biological applications of the graph theory, has highlighted how the brain operates by the interplay amongst the connections of several areas, a process that defines the neural networks in the brain that support 
the human functions in multifarious dynamical and plastic ways Following this line, it is expected that gustatory pathways in the brain might possess peculiar connections related to the genetic profiles of individuals.

The individuals recruited for this study are 5 super-tester and 8 non-testers to the bitter stimulus, as defined by Zhao and coworkers [7] and validated in several studies [8,9]. We examined activations using the four hubs of the Default Mode Network of the brain, defined by Fox et al., as crucial seeds for the connectivity analysis. Our results show the Cortical Areas in which PROP bitter taste signals are differently processed in two genetically different groups (PROP super-tasters and non-taster) consistently with individual differences in the ability to taste this compound. Brain structures and functions are characterized by systems of neurons connections organized in different scale of length [16]. Each system has its own organization, with sub-regions, that have varying degrees of specialization for cognitive and behavioral processes. Studies on brain networking showed a network consisting of a set of functionally interconnected brain regions whose activity decreases during goal-oriented external tasks and increases during quiet waking state [17]. The fMRI signal reflects the change in hemodynamics within a spatial scale in the range of some $\left[\mathrm{mm}^{3}\right]$ brain region volume; this change generates a chain of events defined as neurovascular coupling.

The principal areas of DMN include: Inferior Parietal Cortex (IPC), Posterior Cingulate Cortex (PCC) and Precuneous, Prefrontal Cortex (PFC), Anterior Cingulate Cortex and Medial Temporal Lobe. The network is active in the resting state of the brain with a high degree of functional connectivity between regions; this state has been termed as the default-mode of brain activity to denote a state during which an individual is awake and alert, but not actively involved in an attentional demanding or goal-directed task [18]. The brain's frontoparietal network region is shown to play a central role in routing the process of cognitive tasks among a range of specialized cognitive-processing network hubs which include the visual, auditory, and motor network hubs. Together, our study shows that the areas activated during the taste processing, those involved in processing the risk and possible behavioral conflicts, those related to the inhibition of the response and of the cognitive control of the adaptation, along with those aimed at regulation of blood pressure and heart rhythm which is physiologically modulated by emotions and by the control of basic pulsion. Moreover, in this sketchy account It is crucial to mention the role of the connections to with the areas aimed at evaluating of the risk during the ingestion of food. We should consider that 6-n-Propylthiouracil is a genetic marker for taste, with important implications for food preference and dietary habits.

In other words, the ability to taste the bitter in the Prop could relate to implications for food and drinks preference and dietary habits. Though speculative, it seems likely that our paleoanthropic ancestors would have been equipped with a fine biological sensor in order to discriminate the bitter taste, as an early detection of those often poisonous bitter, substances would have represented a real evolutionary advantage over subjects showing less sensitivity who were more exposed to the toxic action of bitter and poisonous substances. In the economy of this study it seems worth to mention from a neurological point of view is the connection of the MPFC with the BA34 since it is related to the peculiar processing of smell which is in the brain is strictly related to the taste [19]. In addition, the data acquired in our fcMRI experiment suggest an important connection between frontal and parietal networks. Various complex cognitive processes, such as memory acquisition and selection, rely on simultaneous activation of these macro and meso scale brain circuitries. which result not only in broad taste evaluations but also in alloaestetic processes. For instance, the connections of BA33 with the PFC can be referred to a different sensibility to the pain related to the intersubjective variations of the bitter solicitation and at the same time can modulate distinct memory areas whose activation could single out a particular process of pain memorization in the super-tasters.

The results of our study show that fcMRI method can detect and reveal the altered connections of the default mode networks and several functional areas of the brain related to different human genotype in a vital function represented by the taste. Indeed, the bitter taste is differently processed by the two groups of supertaster and non-tester as suggested by the results obtained. However, as the number of our subjects prevents a enough statistical power, it is mandatory to enrol a larger population. Indeed, by increasing the number of the subjects we can predict the possibility that different categories of neural networks could appear in relationship with diverse gustatory pathways as the processing and "interpreting" gustatory information can reveal, at least in part, some creative aspect [20]. As a technical note, our study highlights the importance of using a whole-brain voxel-based measure of functional connectivity, a method that can turn out as a handy exploratory tool for investigating functional changes induced by particular brain states of solicitation as shown by one of the bitter stimuli in particular genotypes. Our results suggest that the voxelbased ICC measures seem to be the optimal approach [21] in order to delineate regions of interest for functional or network analyses. By moving to a voxel level, we are implicitly using a parcellation of the cortex as fine as that allowed in accordance with the acquisition strategy selected.

\section{Conclusion}

The present investigation on the gustatory effects of better taste in a population of subjects represented by super taster and nontaster, lead with the fc-MRI analysis offers a standard framework to perform a large set of connectivity-based experiments on brain functions under a variety of physiological and pathological conditions. Moreover, the calculation of several connectivity 
parameters and measures derived by the graph theory allow for the investigation of brain reactions/responses to diverse pathological and non-pathological conditions. Although the present study illustrated only preliminary data, the results obtained show the reliability of fcMRI investigation, in suggesting that the collaboration between studies on brain networking and "omic" sciences (postgenetic, genomic et al.) is a new exciting approach in exploiting such hard questions as the structural relationships between brain and mind [22].

\section{References}

1. S Bembich, Demarini S, Tepper BJ, Gasparini P, Grasso DL (2014) Individual Differences in Prefrontal Cortex Activity during Perception of Bitter Taste Using fNIRS Methodology. Chem Senses 35(9): 801-812.

2. L Zhao, SV Kirkmeyer, BJ Tepper (2003) A paper screening test to assess genetic taste sensitivity to 6-n-propylthiouracil. Physiol Behav 78(4-5): 625-633.

3. IT Barbarossa, Calò C, Muroni P, Crnjar R, Tepper BJ (2015) The gustin (CA6) gene polymorphism, $\operatorname{rs} 2274333(\mathrm{~A} / \mathrm{G})$, is associated with fungiform papilla density, whereas PROP bitterness is mostly due to TAS2R38 in an ethnically mixed population. Physiol Behav 138: 6-12.

4. G Carta, Melis M, Pintus S, Pintus P (2017) Participants with Normal Weight or with Obesity Show Different Relationships of 6-n-Propylthiouracil (PROP) Taster Status with BMI and Plasma Endocannabinoids. Sci Rep 7(1).

5. KJ Friston (2011) Functional and Effective Connectivity: A Review. Brain Connect 1(1): 13-36.

6. S Whitfield Gabrieli, A Nieto Castanon (2012) Conn: A Functional Connectivity Toolbox for Correlated and Anticorrelated Brain Networks. BRAIN Connect 2(3): 125-141.

7. BJ Tepper (1998) GENETICS OF PERCEPTION '98 6-n-Propylthiouracil: A Genetic Marker for Taste, with Implications for Food Preference and Dietary Habits. Am J Hum Genet. 63(5): 1271-1276.

8. U kyung Kim, E Jorgenson, H Coon, M Leppert, N Risch (2003) Positional cloning of the human quantitative trait locus underlying taste sensitivity to phenylthiocarbamide. Science 299(5610): 1221-1225.

9. B Bufe, Breslin PA, Kuhn C, Reed DR, Tharp CD (2005) The molecular basis of individual differences in phenylthiocarbamide and propylthiouracil bitterness perception. Curr Biol 15(4): 322-327.

\section{ISSN: 2574-1241}

DOI: $10.26717 /$ BJSTR.2020.24.004120

Luigi Barberini. Biomed J Sci \& Tech Res

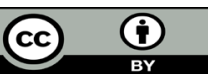

This work is licensed under Creative Commons Attribution 4.0 License

Submission Link: https://biomedres.us/submit-manuscript.php
10.JD Power, Alexander L Cohen, Steven M Nelson, Gagan S Wig (2011) Functional network organization of the human brain. Neuron 72(4): 665-678.

11.G Cossu, Melis M, Sarchioto M, Melis M, Morelli M (2018) 6-n-propylthiouracil taste disruption and TAS2R38 nontasting form in Parkinson's disease. Mov Disord 33(8): 1331-1339.

12. M Melis, L Grzeschuchna, G Sollai, T Hummel, I Tomassini Barbarossa (2019) Taste disorders are partly genetically determined: Role of the TAS2R38 gene, a pilot study. Laryngoscope 129(9): E307-E312.

13. R Martuzzi, R Ramani, M Qiu, X Shen, X Papademetris (2011) A wholebrain voxel-based measure of intrinsic connectivity contrast reveals local changes in tissue connectivity with anesthetic without a priori assumptions on thresholds or regions of interest. Neuroimage 58(4): 1044-1050.

14. MD Greicius, B Krasnow, AL Reiss, V Menon (2003) Functional connectivity in the resting brain: A network analysis of the default mode hypothesis. Proc Natl Acad Sci USA 100(1): 253-258.

15. SF Muldoon, EW Bridgeford, DS Bassett (2016) Small-World Propensity and Weighted Brain Networks. Sci Rep 6: 22057.

16. M Lungarella, O Sporns (2006) Mapping Information Flow in Sensorimotor Networks. PLoS Comput Biol 2(10): e144.

17. ME Raichle, AM MacLeod, AZ Snyder, WJ Powers, DA Gusnard (2001) A default mode of brain function. Proc Natl Acad Sci 98(2): 676-682.

18. AV Utevsky, DV Smith, SA Huettel (2014) Precuneus Is a Functional Core of the Default-Mode Network. J Neurosci 34(3): 932-940.

19. BJ Ciliax, Drash GW, Staley JK, Haber S, Mobley CJ (1999) Immunocytochemical localization of the dopamine transporter in human brain. J Comp Neurol 409(1): 38-56.

20. ER Grimm, NI Steinle (2011) Genetics of eating behavior: established and emerging concepts. Nutr Rev 69(1): 52-60.

21. RL Buckner, Sepulcre J, Talukdar T, Krienen FM, Liu H (2009) Cortical hubs revealed by intrinsic functional connectivity: Mapping, assessment of stability, and relation to Alzheimer's disease. J Neurosci 29(6): 18601873.

22. CL Barberini, SE Morrison, A Saez, B Lau, CD Salzman (2012) Complexity and competition in appetitive and aversive neural circuits. Front Neurosci 6: 170.

23. MJ Domakonda, X He, S Lee, M Cyr, R Marsh (2018) Increased Functional Connectivity Between Ventral Attention and Default Mode Networks in Adolescents with Bulimia Nervosa. 58(2): 232-241.

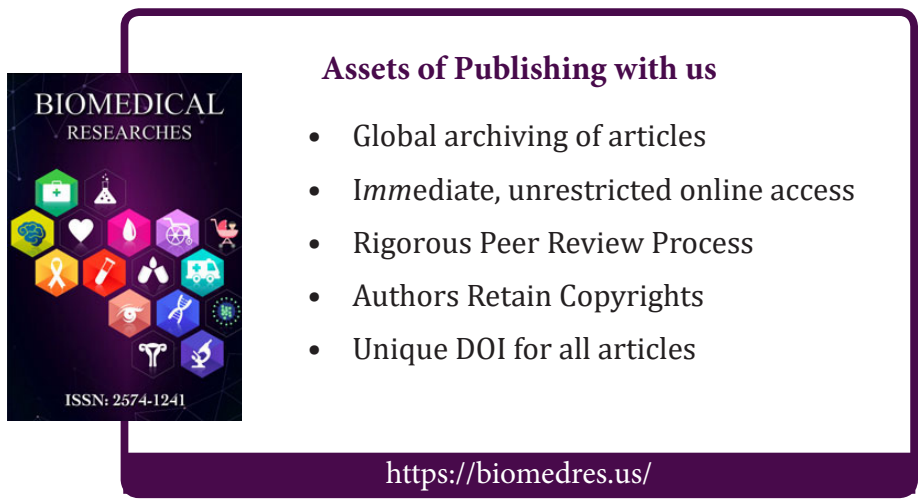

Copyright@ Luigi Barberini | Biomed J Sci \& Tech Res | BJSTR. MS.ID.004120. 